\title{
Angiopoietin-2 (ANGPT2) as a candidate gene for somatic cell score in German Holstein cattle
}

\author{
J. Tetens, ${ }^{* 1}$ C. Baes,†‡ C. Kühn, $\S$ N. Reinsch, $\S$ and G. Thaller* \\ ${ }^{*}$ Christian-Albrechts-University Kiel, Institute of Animal Breeding and Husbandry, 24098 Kiel, Germany \\ †Institute of Agricultural Sciences, Swiss Federal Institute of Technology, 8092 Zürich, Switzerland \\ ¥Qualitas AG, Breeding Division, 6300 Zug, Switzerland \\ §Research Institute for the Biology of Farm Animals, 18196 Dummerstorf, Germany
}

\begin{abstract}
This study evaluated the bovine angiopoietin-2 (ANGPT2) gene as a functional and positional candidate gene underlying a previously fine mapped quantitative trait locus (QTL) for somatic cell score on bovine chromosome 27. The gene product angiopoietin-2 is directly involved in the extravasation of neutrophils, making it a promising candidate to control the amount of somatic cells in milk. A total of 11 polymorphisms were identified within the gene by comparatively resequencing the entire open reading frame of the gene as well as adjacent intronic regions. Four selected variants were genotyped in the same granddaughter design used for QTL mapping and an association study was carried out applying 2 complementary approaches. A variable number of tandem repeats in intron 7 of the gene was found to be significantly associated with cell score in all approaches and explains approximately half of the QTL variance. Analysis of ANGPT2 mRNA expression in different localizations of the udder in animals putatively carrying divergent QTL alleles revealed differential expression in the udder lymph node. Together with the physiological role of angiopoietin-2, these results support a role of $A N G P T 2$ in the genetic control of somatic cell score. Further studies are, however, needed to further underpin this hypothesis.
\end{abstract}

Key words: somatic cell score, angiopoietin-2 (ANGPT2) gene, quantitative trait loci, neutrophil

\section{INTRODUCTION}

In most countries, selection for udder health is based on the indicator trait SCS. We have previously fine mapped a QTL affecting this trait in German Holstein cattle on BTA27, applying a combined linkage and linkage disequilibrium approach (Baes et al., 2010); the

Received March 13, 2013.

Accepted April 14, 2013.

${ }^{1}$ Corresponding author: jtetens@tierzucht.uni-kiel.de
QTL peak is located within the interval between the microsatellite markers KIBS271 and DIK2191 from 6 to $8.5 \mathrm{Mb}$ (Btau 4.0 genome sequence assembly) and encompasses a cluster of $\beta$-defensin genes, which can be regarded as strong candidates (Tetens et al., 2010). However, the angiopoietin-2 (ANGPT2) gene is located at $5.6 \mathrm{Mb}$ in a flanking marker interval, which we have identified as another promising candidate. Its gene product angiopoietin-2 (Ang-2) as well as the related angiopoietin-1 (Ang-1) are ligands of the tyrosine kinase receptor Tie2, which is expressed in endothelial and early hematopoietic cells (Davis et al., 1996; Fiedler et al., 2003); Tie2 and Ang-1 play a crucial role in embryonic angiogenesis (Suri et al., 1996), whereas Ang-2 shares the same receptor binding site (Fiedler et al., 2003) but is an antagonist disrupting in vivo angiogenesis (Maisonpierre et al., 1997). It has been shown that Ang-2 is stored in endothelial cell Weibel-Palade bodies and can be released upon stimulation within minutes, implying the angiopoietin-Tie system in rapid vascular responses (Fiedler et al., 2004). Angiopoietin2-deficient mice fail to elicit an inflammatory response in Staphylococcus aureus-induced peritonitis due to an impaired adhesion of neutrophils to endothelial cells, which can be restored by recombinant Ang-2 (Fiedler et al., 2006). In a transgenic mouse model showing inducible endothelial Ang-2 expression, it was consistently shown that Ang-2 promotes myeloid cell infiltration (Scholz et al., 2011). Several subsequent adhesion processes are necessary to promote the recruitment of neutrophils to inflammatory sites requiring interactions between cell surface molecules of endothelial cells and neutrophils. In this context, angiopoietins have been shown to be capable of promoting the translocation of P-selectin from Weibel-Palade bodies to the surface of endothelial cells (Maliba et al., 2008). The interaction of P-selectin with its counter receptor on neutrophils then mediates their rolling and adhesion (Sako et al., 1993). Angiopoietins were also found to have a chemotactic effect on neutrophils (Sturn et al., 2005; Brkovic et al., 2007) and to directly activate neutrophils and modulate platelet-activating factor synthesis as well as 
functionally upregulate the $\beta_{2}$-integrin complex on the neutrophil surface (Lemieux et al., 2005). The latter binds to endothelial intercellular adhesion molecule (ICAM)-1 and ICAM-2 and increases neutrophil adhesion (Maliba et al., 2008). Together with the fact that Ang-2 expression can be stimulated by bacterial LPS (Mofarrahi et al., 2008), this supports the hypothesis of bovine ANGPT2 being a strong candidate related to udder health and influencing SCS. The aim of the current study was to test for possible associations between bovine ANGPT2 polymorphisms and SCS in our fine-mapping population. Furthermore, the mRNA expression of ANGPT2 at different localizations of the bovine udder was determined in cows presumably carrying divergent QTL alleles to gather evidence for a functional role of ANGPT2 in udder health.

\section{MATERIALS AND METHODS}

\section{Animals and Phenotypes}

For the association study, the same set of animals as in the preceding fine-mapping study (Baes et al., 2010) was used and consisted of 6 paternal half-sib families with a total of 492 genotyped sons from the granddaughter design described by Thomsen et al. (2000). The families were selected based on the QTL-heterozygous state of the grandsires. The average number of sons per sire was 82 and ranged from 28 to 274. Progeny-testing data were available for an average of 190 daughters per sire. Daughter yield deviations (DYD) for SCS obtained from the official release of the German genetic evaluation results (http://www.vit. $\mathrm{de} /$ ?id=zuchtwertschaetzung) were used as phenotypic values. The SCS was calculated from the SCC, applying the formula SCS $=\log _{2}\left(\frac{\mathrm{SCC}}{100,000}\right)+3$. The DYD of the 492 sires ranged from -0.052 to 1.63 , with lower values representing a lower SCC. Daughter yield deviations for the first lactation were used in the analysis, as the number of observations was the highest in this parity.

To address the question of whether ANGPT2 might be differentially expressed between animals with extremely divergent phenotypes, we applied a quantitative real-time $\mathrm{PCR}$ approach to measure ANGPT2 mRNA expression. Therefore, 6 animals with a genetic potential for a low number of somatic cells in milk and 3 animals with the potential for a high number were selected. The first group was assumed to carry primarily QTL alleles decreasing the cell count $(\mathbf{Q})$, whereas the latter group should presumably carry alternative and, thus, unfavorable QTL alleles (q). The selection of those animals was based on the reverse of the rela- tive estimated SCS breeding values of their respective sires, meaning that high breeding values represented the genetic potential for low cell counts. The sires were selected to have extremely high (group Q) and low (group q) breeding values, respectively. The damsires were required to also have congruently high or low breeding values, whereas the dams had to show relative breeding values at least equal to their sires. Using this strategy, animals were selected that could be presumed to primarily carry the favorable (Q) or unfavorable (q) QTL alleles. The procedure was described in detail for a SCS QTL on BTA18 by Kühn et al. (2008).

\section{Polymorphism Screening}

For the detection of polymorphisms within the bovine ANGPT2 gene, PCR products spanning the entire open reading frame and adjacent intronic regions of the gene were amplified from the 8 sires with the lowest and highest DYD values. Polymerase chain reaction primers (Supplemental Table S1, available online at http:// www.journalofdairyscience.org/) were designed based on the bovine reference sequence (RefSeq) mRNA sequence (GenBank NM_001098855.1; http://www.ncbi. nlm.nih.gov/genbank/) aligned to genome build Btau 4.0 (GenBank NC_007328.3), applying the web-based primer design tool Primer3 (http://frodo.wi.mit.edu/ primer3/). The PCR reactions were carried out in a final volume of $12 \mu \mathrm{L}$ using a standard Taq DNA polymerase (Invitek GmbH, Berlin, Germany) together with the buffer supplied by the manufacturer and a final $\mathrm{Mg}^{2+}$ concentration of $1.5 \mathrm{~m} M$. The subsequent resequencing of the PCR products was performed after rAPid alkaline phosphatase (Roche Diagnostics GmbH, Mannheim, Germany) and exonuclease I (New England BioLabs Inc., Ipswich, MA) treatment using both PCR primers with the ABI BigDye Terminator Sequencing Kit 3.1 (Applied Biosystems, Darmstadt, Germany) on an ABI 3130xl capillary sequencer (Applied Biosystems). Internal sequencing primers were used for the PCR-products spanning exons 6 and 7 (Supplemental Table S1, available online at http://www.journalof dairyscience.org/). Sequence data were analyzed using the Sequencher 4.9 software (Gene Codes Corp., Ann Arbor, MI).

\section{Association Analysis}

Four polymorphisms [1 SNP in intron 1 (SNP 3$), 1$ SNP in exon 3 (SNP 4), 1 variable number of tandem repeats (VNTR) intron 7 , and 1 SNP within the $3^{\prime}$ untranslated region (SNP 10); see Table 1 and Figure 1] were selected for genotyping in the 492 sons of the granddaughter design. The selection criteria were the 
position within the gene, technical suitability for genotyping, and a differential allelic distribution between the 2 extreme groups used for comparative resequencing. The VNTR was genotyped by amplification of a 142-bp PCR product with a labeled forward primer (Supplemental Table S1, available online at http:// www.journalofdairyscience.org/) and subsequent fragment length analysis on an ABI 3130xl capillary sequencer. The SNP 3 and 10 (Table 1 and Figure 1) were genotyped by restriction endonuclease digestion of the original PCR products (Ex1b and Ex9c; Supplemental Table S1), applying the enzymes HpyCH4IV and Tth111I (New England BioLabs Inc.) and subsequent fragment length analysis on a $2 \%$ agarose gel. The conditions and resulting fragments are given in Table 1. Single nucleotide polymorphism 4 was genotyped using the tetra-primer amplification refractory mutation system (ARMS)-PCR approach according to Ye et al. (2001; see Table 1 and Supplemental Table S1). The primers were designed with the web tool BatchPrimer3 (You et al., 2008; http://probes.pw.usda.gov/ batchprimer3/index.html). The PCR was carried out using the Qiagen GmbH (Hilden, Germany) Multiplex PCR Kit according to the manufacturer's instructions in a final volume of $12 \mu \mathrm{L}$, containing $0.1 \mu \mathrm{M}$ concentration of each outer and $1 \mu M$ concentration of each allele-specific inner primer. The PCR products were separated on a $3 \%$ agarose gel.

Statistical analyses were carried out using the $\mathrm{R}$ environment (R Core Team, 2012) with the packages car (Fox and Weisberg, 2011) and genetics (Warnes and Leisch, 2012). Linkage disequilibrium (LD) between the genotyped markers was calculated as the squared correlation $\left(\mathrm{r}^{2}\right)$. Single marker association analysis was performed applying the following weighted linear regression model:

$$
y_{i j}=\mu+b \cdot \mathrm{SNP}+s_{i}+e_{i j}
$$

where $y_{i j}$ is the DYD of son $j$ within sire $i, \mu$ is the overall mean, $b$ is the regression coefficient representing half of the allele substitution effect $(\alpha / 2$; Falconer and Mackay, 1996), SNP represents the genotype, $s_{i}$ is the fixed effect of sire $\mathrm{i}$, and $e_{i j}$ is the random residual effect. Marker genotypes were coded as 0 (homozygous for the rare allele), 1 (heterozygous), and 2 (homozygous for the alternative allele). The DYD were weighted by the effective daughter contributions following Liu et al. (2004). Multimarker analyses were carried out with an analogous model but fitting all marker effects at the same time. The proportion of phenotypic variance jointly explained by these markers was estimated from the residual sum of squares (RSS) of this model (full model) and a second model omitting the marker effects (reduced model) according to the following formula:

$$
\text { Explained variance }=\frac{\mathrm{RSS}_{\text {reduced model }}-\mathrm{RSS}_{\text {full model }}}{\operatorname{RSS}_{\text {reduced model }}} \cdot 100 .
$$

In a complementary approach, each marker was included as a fixed effect into the model applied to the data for across-family linkage analysis in the preceding fine-mapping study. This study was conducted using 18 microsatellite markers spanning approximately 26 $\mathrm{cM}$ at the centromeric end of BTA27. The construction of the linkage map and marker details can be found in Baes et al. (2010); Figure 2 summarizes the marker positions, where the midpoint of each marker interval was considered as a putative QTL position. The statistical procedures used for QTL mapping have been extensively described in Baes et al. (2009), Baes et al. (2010), and Baes and Reinsch (2008). Briefly, the following model containing a random polygenic effect as well as a random additive QTL effect (Baes et al., 2009) was applied to the data for across-family linkage analysis:

$$
\mathbf{y}=\mathbf{X} \boldsymbol{\beta}+\mathbf{Z u}+\mathbf{W} \mathbf{v}+\mathbf{e},
$$

where $\mathbf{y}$ is a vector of DYD; $\mathbf{X}$ is a design matrix; $\boldsymbol{\beta}$ is a vector of fixed effects common to all animals; $\mathbf{Z}$ is an incidence matrix relating animals to phenotypes; $\mathbf{u}$ is a vector of random polygenic effects, with $\mathbf{u} \sim N\left(0, \mathbf{A}_{p} \sigma_{p}^{2}\right)$; $\mathbf{W}$ is an incidence matrix; $\mathbf{v}$ is a vector of random QTL effects, with $\mathbf{v} \sim N\left(0, \mathbf{G}_{q} \sigma_{q}^{2}\right)$; and $\mathbf{e}$ is a residual vector, with $\mathbf{e} \sim N\left(0, \mathbf{D} \sigma_{e}^{2}\right)$. The variances $\sigma_{p}^{2}, \sigma_{q}^{2}$, and $\sigma_{e}^{2}$ denote the polygenic, the additive QTL, and the residual variance, respectively; $\mathbf{A}_{p}$ is the additive genetic relationship matrix; $\mathbf{G}_{q}$ is the (co)variance matrix for the additive effects of the QTL conditional on marker haplotypes at a given position (Fernando and Grossman, 1989); and the diagonal matrix $\mathbf{D}$ contains 1 over the effective daughter contributions (Liu et al., 2004). The restricted log-likelihood ratio test (RLRT) statistic for each putative QTL position (RLRT ${ }^{\mathrm{Pos}}$ ) was calculated as follows:

$$
\operatorname{RLRT}^{\text {Pos }}=-2\left[\ln \left(L_{0}\right)-\ln \left(L_{1}^{\text {Pos }}\right)\right],
$$

where $L_{1}^{P o s}$ is the RLRT of model 1 for position Pos and is that of the same model without random gametic effects for a given QTL. Test statistics were calculated using ASReml software (Gilmour et al., 2009) within the TIGER software system (Baes and Reinsch, 2008). 
Table 1. Polymorphisms within the bovine angiopoietin-2 (ANGPT2) gene detected by comparative resequencing ${ }^{1}$

\begin{tabular}{|c|c|c|c|c|c|}
\hline $\begin{array}{l}\text { SNP } \\
\text { identification }\end{array}$ & Location $^{2}$ & Description $^{3}$ & $\mathrm{SNPdb}^{4}$ & $\begin{array}{l}\text { Genomic } \\
\text { coordinate }^{5}\end{array}$ & $\begin{array}{l}\text { Genotyping } \\
\text { details }^{6}\end{array}$ \\
\hline ANGPT2_SNP1 & Intron 1 & ANGPT2_c. $291+64 \mathrm{C}>\mathrm{T}$ & rs43706125 & $5,682,412$ & \\
\hline ANGPT2_SNP2 & Intron 1 & ANGPT2_c.292-192A > G & None & $5,652,984$ & \\
\hline ANGPT2_SNP3 & Intron 1 & ANGPT2_c.292-100A $>$ G & None & $5,652,892$ & $\begin{array}{l}\text { RFLP } \\
\text { Enzyme: HpyCH4IV } \\
\text { Incubation: } 65^{\circ} \mathrm{C} / 2 \mathrm{~h} \\
\text { Fragments: } \\
\text { A: } 691 \mathrm{bp} \text { (uncut) } \\
\text { G: } 369 / 322 \mathrm{bp}\end{array}$ \\
\hline ANGPT2_SNP4 & Exon 3 & ANGPT2_c.498C $>\mathrm{T}$ & None & $5,622,162$ & $\begin{array}{l}\text { Tetra-primer-ARMS } \\
\text { Outer product: } 334 \mathrm{bp} \\
\text { Inner product T: } 171 \mathrm{bp} \\
\text { Inner product C: } 203 \mathrm{bp}\end{array}$ \\
\hline ANGPT2_SNP5 & Intron 3 & ANGPT2_c.569+13C > T & None & $5,622,078$ & \\
\hline$A N G P T 2 \_V N T R$ & Intron 7 & ANGPT2_c. $1199+85$ TCAGA $\left(3 \_4\right)$ & None & $5,606,839$ & Fragment length analysis \\
\hline ANGPT2_SNP6 & Intron 8 & ANGPT2_c.1331-193C > T & None & $5,599,506$ & \\
\hline ANGPT2_SNP7 & Intron 8 & ANGPT2_c.1331-145A > G & None & $5,599,458$ & \\
\hline ANGPT2 SNP8 & Intron 8 & ANGPT2_c.1331-52A > C & None & $5,599,365$ & \\
\hline ANGPT2_SNP9 & Exon 9 & ANGPT2_c. $1461 \mathrm{~A}>\mathrm{G}$ & None & $5,599,183$ & \\
\hline ANGPT2_SNP10 & Exon $9 / 3^{\prime}$-UTR & ANGPT2_c. $* 28 \mathrm{C}>\mathrm{T}$ & None & $5,599,125$ & $\begin{array}{l}\text { RFLP } \\
\text { Enzyme: Tth111I } \\
\text { Incubation: } 37^{\circ} \mathrm{C} / 2 \mathrm{~h} \\
\text { Fragments: } \\
\text { T: } 591 \mathrm{bp} \text { (uncut) } \\
\text { C: } 372 / 219 \mathrm{bp}\end{array}$ \\
\hline
\end{tabular}

Polymorphisms genotyped for the association analysis are printed in boldface.

${ }^{2} \mathrm{UTR}=$ untranslated region.

${ }^{3}$ Positions are given according to reference sequence (RefSeq) GenBank NM_001098855.1 (http://www.ncbi.nlm.nih.gov/genbank/).

${ }^{4}$ Identifier in the single nucleotide polymorphism database (http://www.ncbi.nlm.nih.gov/snp).

${ }^{5}$ According to bovine genome assembly Btau 4.0 (GenBank NC_007328.3).

ARMS = amplification refractory mutation system 


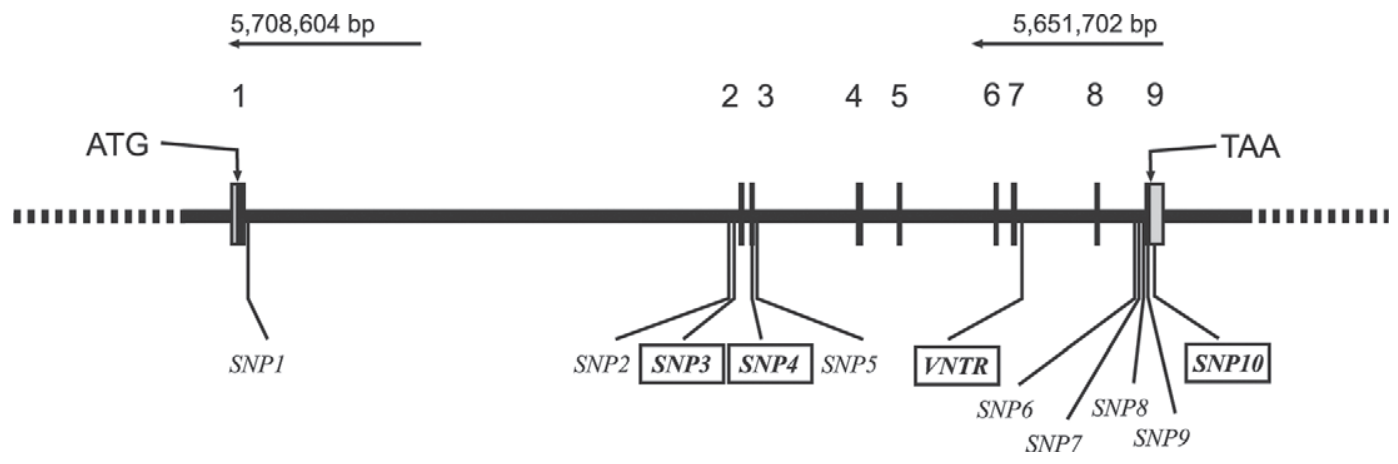

Figure 1. Structure of the bovine angiopoietin-2 (ANGPT2) gene based on the alignment of the reference mRNA sequence (GenBank NM_001098855.1; http://www.ncbi.nlm.nih.gov/genbank/) to the Btau 4.0 genome assembly and positions of the polymorphisms detected by comparative resequencing. Black and gray boxes indicate exonic regions belonging to the open reading frame and the untranslated regions, respectively. Exon numbering is given in Arabic numerals. Details regarding the polymorphisms can be found in Table 1. Polymorphisms selected for the association study are highlighted by black boxes. ATG = start codon; TAA = stop codon; VNTR = variable number of tandem repeats.

To test for significant association with SCS, candidate polymorphisms were included as fixed effects into the linkage-mapping model. The proportion of QTL and polygenic variance explained by the polymorphism was calculated using the estimated variance components with a fixed marker effect at the QTL peak position and a reduced model without marker effect.

As 4 markers were tested in all association analyses, the results were adjusted for multiple testing. Due to linkage disequilibrium between the markers, the tests are, however, not independent. Thus, the approach according to Cheverud (2001) was applied, where the number of independent tests is calculated based on the correlation between markers following the following formula:

$$
M_{e f f}=M\left[1-(M-1) \frac{\sigma_{\lambda o b s}^{2}}{M^{2}}\right],
$$

where $M_{\text {eff }}$ and $M$ are the effective and the original number of markers, respectively, and $\sigma_{\text {tobs }}^{2}$ is the observed variance of the eigenvalues derived from the correlation matrix of the additive marker genotypes. The number of effective markers, which was calculated as 2.3 in the current study, was used in a less stringent Bonferroni correction procedure.

\section{Expression Analysis}

For each animal, tissue samples from the streak canal, the rosette of Fürstenberg, the milk cistern, the udder lymph node and the udder parenchyma were available. Sample collection and preparation have been described in Tetens et al. (2010).

Quantitative real-time PCR primers (Supplemental Table S1, available online at http://www.journalof dairyscience.org/) were designed using the Primer3 software (http://frodo.wi.mit.edu/primer3/) and synthesized by biomers.net (Ulm, Germany). Based on the bovine ANGPT2 reference mRNA sequence (GenBank NM_001098855.1) aligned to the Btau 4.0 genome assembly, 3 primer pairs were designed spanning different regions of the mRNA (see Supplemental Table S1). One primer pair was located in exon 1 and 2 were spanning the boundaries between exons 1 and 2 and exons 8 and 9, respectively. An additional primer pair spanning the region from exons 4 to 8 and a primer pair for the reference gene $\beta$-actin were adopted from Hayashi et al. (2004). The quantitative real-time PCR was carried out on an iQ5 Multicolor Real-Time PCR Detection System (Bio-Rad Laboratories GmbH, Munich, Germany). The reactions were set up in a final volume of $20 \mu \mathrm{L}$ containing $0.4 \mu \mathrm{M}$ concentration of each primer using the Platinum SYBR Green quantitative PCR (qPCR) Super Mix UDG Kit (Invitrogen Corp., Carlsbad, CA). The amplification was done using $2 \mu \mathrm{L}$ of template cDNA, applying the following protocol: after an initial denaturation at $95^{\circ} \mathrm{C}, 40$ cycles were performed consisting of denaturation $\left(95^{\circ} \mathrm{C}\right.$ for $\left.15 \mathrm{~s}\right)$, annealing $\left(58^{\circ} \mathrm{C}\right.$ for $30 \mathrm{~s})$, elongation $\left(72^{\circ} \mathrm{C}\right.$ for $\left.30 \mathrm{~s}\right)$, and a high-temperature fluorescence acquisition step $\left(81\right.$ or $85^{\circ} \mathrm{C}$ for $30 \mathrm{~s}$; see Supplemental Table S1, available online at http://www. journalofdairyscience.org/; Pfaffl, 2001). This protocol was followed by a melting curve analysis. The threshold cycle $\left(\mathbf{C}_{t}\right)$ of a sample was set at the cycle number at which the fluorescence signal exceeded the background noise, as automatically determined by the iQ5 Optical System Software (Bio-Rad Laboratories GmbH). All reactions were done in triplicate and all samples were run simultaneously with the target and the reference gene primers. The triplicate measures were checked for uniformity using the outlier test according to Nalimov (1963) and the mean of the 3 measurements was used for further calculations. 
The quantification was done by applying the $\Delta C_{t}$ method, where $\Delta \mathrm{C}_{\mathrm{t}}$ for each sample is given by the difference between the $\mathrm{C}_{t}$ values of the target and the reference gene and the relative mRNA expression is then calculated as $2^{-\Delta \mathrm{C}_{\mathrm{t}}}$. Based on the high correlation between the relative expression values calculated from 3 PCR products (exon 1, exons 1 and 2, and exons 4 to 8), the relative ANGPT2 mRNA expression (RE) was calculated for each sample as the mean of the 3 assays according to the following formula:

$\mathrm{RE}=$

$2^{-\left\{\frac{1}{3}\left[C_{t}\left(A N G P T 2_{E x_{-}}\right)+C_{t}\left(A N G P T 2_{E x_{-}} 1 / 2\right)+C_{t}\left(A N G P T 2_{E x_{-}} 4-8\right)\right]-C_{t}(B A C T)\right\}}$,

where $C_{t}\left(A N G P T 2_{E x_{-} x}\right)$ is the $\mathrm{C}_{\mathrm{t}}$ value determined for the respective PCR product of ANGPT2 (Supplemental Table S1, available online at http://www. journalofdairyscience.org/), with Ex representing exon, and $C_{t}(B A C T)$ is the corresponding $\mathrm{C}_{\mathrm{t}}$ value for the reference gene $\beta$-actin. The fourth PCR product (exons 8/9) was analyzed separately.

\section{RESULTS}

\section{Detection of Polymorphisms}

We resequenced approximately $5 \mathrm{~kb}$ of the bovine $A N G P T 2$ gene covering the entire open reading frame and adjacent intronic regions in 16 animals. Eleven polymorphisms comprising $10 \mathrm{SNP}$ and a VNTR were detected (Figure 1; Table1). Two synonymous transitions were found at positions 498 (exon 3) and 1,461 (exon 9) of the open reading frame and one $28 \mathrm{bp}$ from the stop codon within the $3^{\prime}$ untranslated region. The remaining polymorphisms were located in introns (Figure 1; Table1). One SNP in intron 1 (SNP 3) and 2 SNP in exon 3 (SNP 4) and exon 9 (SNP 10), respectively, as well as the VNTR in intron 7 were selected for the association analysis.

\section{Association Analysis}

The number of animals genotyped for each of the 4 markers ranged from 482 to 490 (Table 2), with 481 animals successfully genotyped at all 4 loci. Three markers, the VNTR, and the SNP 3 and 4 in intron 1 and exon 3 , respectively, showed significant associations with SCS, with adjusted $P$-values $\leq 0.01$ in single-marker regression. These markers showed a pairwise LD of 0.91 (VNTR vs. SNP 3), 0.99 (VNTR vs. SNP 4), and 0.90 (SNP 3 vs. SNP 4). Single nucleotide polymorphism 10 was not significantly associated and in moderate LD with the other markers showing squared correla-

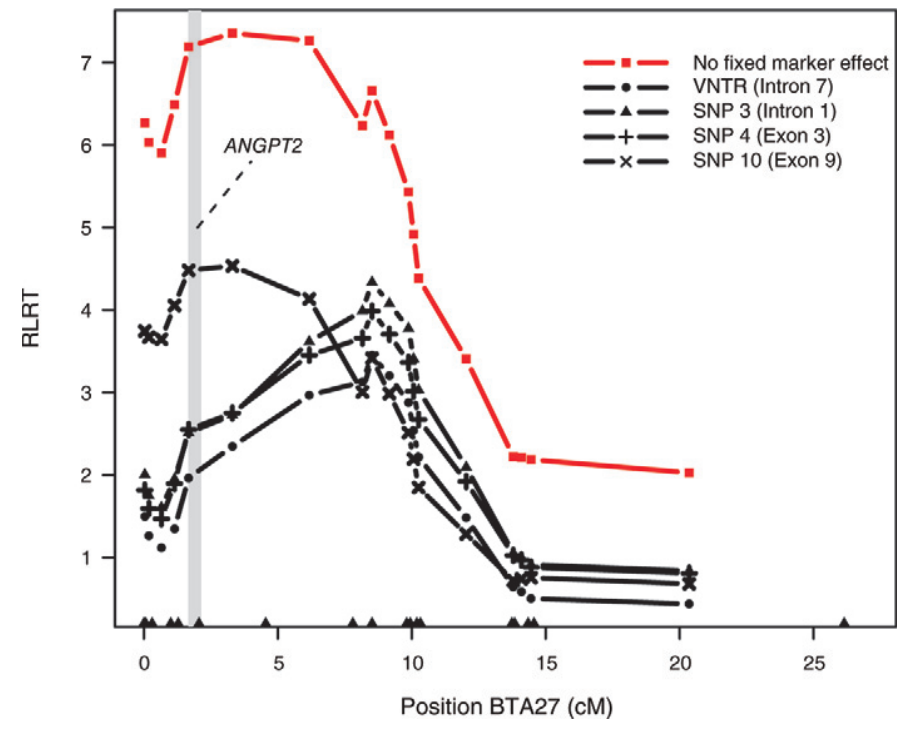

Figure 2. Profiles of the restricted log-likelihood ratio test (RLRT) statistics as obtained from the model used for across-family linkage analysis. The red line (with squares) represents the original test statistic without the inclusion of any fixed marker effects. The black curves (all curves except for that with squares) show the results obtained when including the different markers. Except for SNP 10 (not significant), the significant markers alter the RLRT profile in a similar way. Triangles on the abscissa indicate the positions of the microsatellites used for mapping (Baes et al., 2010). VNTR = variable number of tandem repeats. BTA27 = bovine chromosome 27. Color version available in the online PDF.

tion $\left(\mathrm{r}^{2}\right)$ values of 0.56 (VNTR), 0.48 (SNP 3), and 0.54 (SNP 4). The lowest adjusted $P$-value of 0.00016 was obtained for the VNTR and $\alpha / 2$ was estimated as $0.168 \pm 0.04$ for this marker (Table 2). In multimarker regression, this was the only marker showing a marginally significant association, with an adjusted $P$-value of 0.062 (nominal $P$-value $=0.027$ ) and an estimated effect of $0.421 \pm 0.19$. The proportion of DYD variance jointly explained by the markers in multimarker regression was calculated as $6.46 \%$.

The inclusion of each marker in the linkage mapping model revealed effects for the same markers shown to be associated in single-marker regression (i.e., the VNTR and SNP 3 and 4; Table 2). The lowest adjusted $P$-value of 0.014 was consistently obtained for the VNTR, whereas SNP 3 and SNP 4 were only marginally significant, with adjusted $P$-values $\leq 0.1$. Comparing the likelihood profile of the original linkage mapping [red curve (with squares) in Figure 2] with those obtained by the inclusion of fixed candidate marker effects [black curves (all curves except that with squares) in Figure 2] reveals the strong impact of the associated markers, especially within the proximal region containing the QTL peak. The distal part is, though on a lower RLRT level, unchanged. Single nucleotide polymorphism 10, which is not significantly associated, does 
not exhibit this effect on the likelihood profile. From the model without the inclusion of any candidate marker effects, a polygenic variance $\left(\sigma_{p}^{2}\right)$ of 0.163 was estimated for the peaking marker interval. The QTL variance at this position, calculated as twice the gametic variance, is $\sigma_{q}^{2}=0.025$; thus, the ratio of QTL to polygenic variance is 0.153 , meaning that the QTL accounts for approximately $15 \%$ of the genetic variance for SCS. Based on the estimated variances from the models including fixed marker effects, it can be calculated that the significantly associated markers explain approximately half of the QTL variance (Table 2), corresponding to approximately $7.5 \%$ of the genetic variance.

\section{mRNA Expression Analysis}

The relative expression values were calculated separately for each PCR product and checked for pair-wise correlation. The values calculated from the primer pairs located in exon 1 , exons $1 / 2$ and exons $4 / 8$ (see Supplemental Table S1, available online at http://www. journalofdairyscience.org/) were found to be highly correlated with each other, showing correlation coefficients $>0.94$. The values calculated from the primer pair located in exons 8 and 9 were less correlated with the other measures, especially in the rosette of Fürstenberg and the lymph node, with correlation coefficients of around 0.7. Based on this observation, the relative expression was calculated as the mean of the highly correlated values and the PCR product for exon 8/9 was analyzed separately.

The highest relative ANGPT2 expression with respect to the combined analysis of exons 1, 1 and 2, and 4 to 8 was found in the cistern, followed by the udder parenchyma (Figure 3), whereas the overall expression level in the other tissues was on a similarly low level. No significant differences were found between the 2 divergent groups, except for the lymph node. The relative expression in this tissue was significantly $(P=0.023)$ lower in the $\mathrm{Q}$ than in the q group. No significant differences were found for the remaining PCR product corresponding to exon $8 / 9$.

\section{DISCUSSION}

Within the current study, ANGPT2 was evaluated as a possible candidate gene affecting SCS in Holstein dairy cattle. The gene is located within a previously fine-mapped QTL region for this trait (Baes et al., 2010). Although an adjacent cluster of $\beta$-defensin genes also represents strong candidates (Tetens et al., 2010), the molecular function of Ang-2, the gene product of 


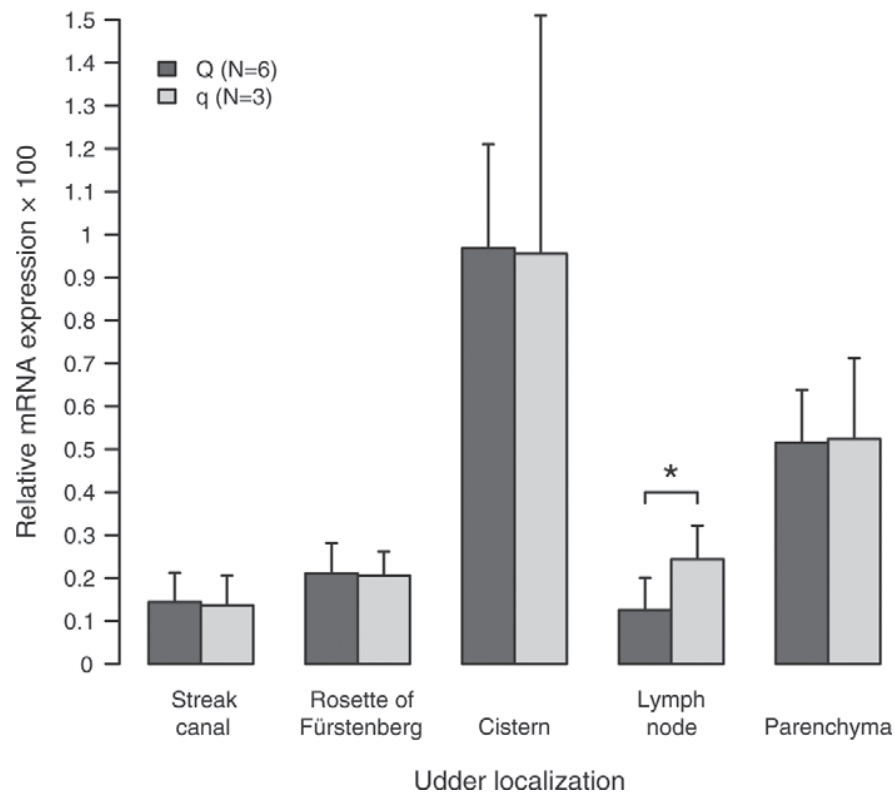

Figure 3. Relative angiopoietin-2 (ANGPT2) mRNA expression in different localizations of the udder of animals presumably carrying divergent QTL alleles (dark gray $=\mathrm{Q}=$ low cell count; light gray $=$ $\mathrm{q}=$ high cell count). Error bars depict the respective SE; significant differences $(P \leq 0.05)$ are indicated by an asterisk.

ANGPT2, predisposes this gene to affect SCS. Angiopoietin-2 is directly involved in the extravasation of neutrophils (Maliba et al., 2008; Scholz et al., 2011). Thus, it partly controls the number of somatic cells leaving the bloodstream and appearing, for example, in udder tissue and milk.

The association analysis conducted in the current study revealed significant associations with SCS for 3 of the tested markers. Results of the 2 complementary approaches were consistent. The allele substitution effects of the 3 significantly associated markers obtained from single-marker regression were similar in size and collectively explained approximately half of the QTL variance when included in the linkage mapping model. Furthermore, the effects on the likelihood profiles (Figure 2) were almost identical among these markers, reflecting the high pairwise LD between these markers. However, the VNTR showed the strongest association and was the only significant marker in multimarker regression exhibiting an allele substitution effect of $\alpha$ $=0.84$.

In most localizations of the udder, no differential Ang-2 mRNA expression was observed between groups of cows presumably carrying divergent QTL alleles, except for the udder lymph node (Figure 3). The q group showed a higher Ang-2 mRNA-expression, meaning that these animals should exhibit higher cell counts. This is consistent with the QTL-mapping results, be- cause the preferable Q allele indicates lower cell counts and might indicate a functional role of $A N G P T 2$ in the control of SCC. A further noteworthy result of the expression analysis was the lower correlation between the PCR product spanning exons 8 and 9 and the other PCR products, which may indicate the presence of alternative splicing involving exon 9. However, the expression results are based on a small sample size and should be repeated using more comprehensive animal material. Furthermore, the analyzed cows were healthy and no data exist regarding Ang-2 expression in case of mammary infection. Assuming, however, a functional role of ANGPT2 implicates the risk of partly impairing local mammary host defense by selection solely based on SCC. Several studies have reported high genetic correlations between cell count and clinical mastitis of approximately 0.7 (Kadarmideen and Pryce, 2001; Rupp and Boichard, 2001; Koeck et al., 2010). This correlation is based on the fact that mammary infection results in elevated cell counts. Selecting for a low cell count improves udder health due to a correlated selection response toward enhanced host defense, preventing clinical mastitis. Local host defense might, however, not be the only physiological target of selection toward low cell counts. In the case of Ang-2, the cell count would be directly affected and the ability to respond to udder infections by the extravasation of neutrophils could be impaired.

The most significantly associated polymorphism identified within this study explained approximately half of the QTL variance. Thus, it may be assumed that it is not the causal variant. The causal variant could possibly reside in the promoter region, which was not analyzed in the current study. On the other hand, it cannot be excluded that the QTL in fact represents 2 closely linked QTL arising from causal genetic variation in ANGPT2 as well as in the adjacent defensin cluster, which has also been linked to udder health (Tetens et al., 2010). The identification of putative causal variation within the defensin cluster is, however, hampered by the fact that the different defensins exhibit a very high degree of sequence homology among one another and show extensive copy number variation (Liu et al., 2010; Hou et al., 2011; Bickhart et al., 2012). Within the current study, only synonymous mutations were identified within the open reading frame of ANGPT2. Together with the fundamental physiological role in immune response and angiogenesis and the fact we observed differential expression at least in the lymph node, this leads to the presumption that putative causal variation for SCC within this gene is most likely represented by a regulatory mutation. Whether the observed VNTR in intron 7 has a regulatory role and affects ANGPT2 expression cannot be evaluated from the existing data. 


\section{CONCLUSIONS}

We showed that polymorphisms within the bovine ANGPT2 gene were significantly associated with SCS in a large granddaughter design. The gene is located in a previously fine-mapped QTL interval on BTA27. Differential mRNA expression in the udder lymph nodes of cows presumably carrying divergent QTL alleles was demonstrated. Together with the essential function of the gene product Ang-2 in the extravasation of neutrophils, our findings back up the hypothesis that genetic variation in ANGPT2 affects SCC in dairy cattle, implicating a possible impairment of host defense through selection for low cell counts. The closely linked $\beta$-defensin cluster on BTA27 might, however, carry additional causative genetic variation. The role of ANGPT2 in SCC in dairy cattle has to be confirmed and further elucidated.

\section{ACKNOWLEDGMENTS}

We are grateful to the German Federal Ministry of Education and Research (Bonn, Germany) for funding the project M.A.S.Net within the FUGATO framework (grant no. 0313390D). Furthermore, we thank the German genetic evaluation center Vereinigte Informationssysteme Tierhaltung (VIT) in Verden for their support with data.

\section{REFERENCES}

Baes, C., B. Brand, M. Mayer, C. Kühn, Z. Liu, F. Reinhardt, and N. Reinsch. 2009. Refined positioning of a quantitative trait locus affecting somatic cell score on chromosome 18 in the German Holstein using linkage disequilibrium. J. Dairy Sci. 92:4046-4054.

Baes, C., M. Mayer, J. Tetens, Z. Liu, F. Reinhardt, G. Thaller, and N. Reinsch. 2010. Refined mapping of a QTL for somatic cell score on BTA27 in the German Holstein using combined linkage and linkage disequilibrium analysis. Can. J. Anim. Sci. 90:169-178.

Baes, C., and N. Reinsch. 2008. TIGER: A software system for finemapping quantitative trait loci. Arch. Tierzucht 51:402-412.

Bickhart, D. M., Y. Hou, S. G. Schroeder, C. Alkan, M. F. Cardone, L. K. Matukumalli, J. Song, R. D. Schnabel, M. Ventura, J. F. Taylor, J. F. Garcia, C. P. Van Tassell, T. S. Sonstegard, E. E. Eichler, and G. E. Liu. 2012. Copy number variation of individual cattle genomes using next-generation sequencing. Genome Res. 22:778-790.

Brkovic, A., M. Pelletier, D. Girard, and M. G. Sirois. 2007. Angiopoietin chemotactic activities on neutrophils are regulated by PI-3K activation. J. Leukoc. Biol. 81:1093-1101.

Cheverud, J. M. 2001. A simple correction for multiple comparisons in interval mapping genome scans. Heredity 87:52-58.

Davis, S., T. H. Aldrich, P. F. Jones, A. Acheson, D. L. Compton, V. Jain, T. E. Ryan, J. Bruno, C. Radziejewski, P. C. Maisonpierre, and G. D. Yancopoulos. 1996. Isolation of angiopoietin-1, a ligand for the TIE2 receptor, by secretion-trap expression cloning. Cell $87: 1161-1169$.

Falconer, D. S., and T. F. C. Mackay. 1996. Introduction to Quantitative Genetics. 4th ed. Pearson Education Ltd., Essex, UK.

Fernando, R. L., and M. Grossman. 1989. Marker assisted selection using best linear unbiased prediction. Genet. Sel. Evol. 21:467-477.
Fiedler, U., T. Krissl, S. Koidl, C. Weiss, T. Koblizek, U. Deutsch, G. Martiny-Baron, D. Marmé, and H. G. Augustin. 2003. Angiopoietin- 1 and angiopoietin- 2 share the same binding domains in the Tie-2 receptor involving the first Ig-like loop and the epidermal growth factor-like repeats. J. Biol. Chem. 278:1721-1727.

Fiedler, U., Y. Reiss, M. Scharpfenecker, V. Grunow, S. Koidl, G. Thurston, N. W. Gale, M. Witzenrath, S. Rosseau, N. Suttorp, A. Sobke, M. Herrmann, K. T. Preissner, P. Vajkoczy, and H. G. Augustin. 2006. Angiopoietin-2 sensitizes endothelial cells to TNFalpha and has a crucial role in the induction of inflammation. Nat. Med. 12:235-239.

Fiedler, U., M. Scharpfenecker, S. Koidl, A. Hegen, V. Grunow, J. M. Schmidt, W. Kriz, G. Thurston, and H. G. Augustin. 2004. The Tie-2 ligand angiopoietin-2 is stored in and rapidly released upon stimulation from endothelial cell Weibel-Palade bodies. Blood 103:4150-4156.

Fox, J., and S. Weisberg. 2011. An R Companion to Applied Regression. 2nd ed. Sage, Thousand Oaks, CA.

Gilmour, A. R., B. J. Gogel, B. R. Cullis, and R. Thompson,. 2009. ASReml User Guide. Release 3.0. VSN International Ltd., Hemel Hempstead, UK.

Hayashi, K.-G., B. Berisha, M. Matsui, D. Schams, and A. Miyamoto. 2004. Expression of mRNA for the angiopoietin-Tie system in granulosa cells during follicular development in cows. J. Reprod. Dev. 50:477-480.

Hou, Y., G. E. Liu, D. M. Bickhart, M. F. Cardone, K. Wang, E. Kim, L. K. Matukumalli, M. Ventura, J. Song, P. M. VanRaden, T. S. Sonstegard, and C. P. Van Tassell. 2011. Genomic characteristics of cattle copy number variations. BMC Genomics 12:127.

Kadarmideen, H. N., and J. E. Pryce. 2001. Genetic and economic relationships between somatic cell count and clinical mastitis and their use in selection for mastitis resistance in dairy cattle. Anim. Sci. 73:19-28.

Koeck, A., B. Heringstad, C. Egger-Danner, C. Fuerst, P. Winter, and B. Fuerst-Waltl. 2010. Genetic analysis of clinical mastitis and somatic cell count traits in Austrian Fleckvieh cows. J. Dairy Sci. 93:5987-5995.

Kühn, C., F. Reinhardt, and M. Schwerin. 2008. Marker assisted selection of heifers improved milk somatic cell count compared to selection on conventional pedigree breeding values. Arch. Tierzucht $51: 23-38$.

Lemieux, C., R. Maliba, J. Favier, J.-F. Théorêt, Y. Merhi, and M. G. Sirois. 2005. Angiopoietins can directly activate endothelial cells and neutrophils to promote proinflammatory responses. Blood 105:1523-1530.

Liu, G. E., Y. Hou, B. Zhu, M. F. Cardone, L. Jiang, A. Cellamare, A. Mitra, L. J. Alexander, L. L. Coutinho, M. E. Dell'Aquila, L. C. Gasbarre, G. Lacalandra, R. W. Li, L. K. Matukumalli, D. Nonneman, L. C. A. Regitano, T. P. L. Smith, J. Song, T. S Sonstegard, C. P. Van Tassell, M. Ventura, E. E. Eichler, T. G. McDaneld, and J. W. Keele. 2010. Analysis of copy number variations among diverse cattle breeds. Genome Res. 20:693-703.

Liu, Z., F. Reinhardt, A. Bunger, and R. Reents. 2004. Derivation and calculation of approximate reliabilities and daughter yield-deviations of a random regression test-day model for genetic evaluation of dairy cattle. J. Dairy Sci. 87:1896-1907.

Maisonpierre, P. C., C. Suri, P. F. Jones, S. Bartunkova, S. J. Wiegand, C. Radziejewski, D. Compton, J. McClain, T. H. Aldrich, N Papadopoulos, T. J. Daly, S. Davis, T. N. Sato, and G. D. Yancopoulos. 1997. Angiopoietin-2, a natural antagonist for Tie2 that disrupts in vivo angiogenesis. Science 277:55-60.

Maliba, R., A. Brkovic, P. E. Neagoe, L. R. Villeneuve, and M. G. Sirois. 2008. Angiopoietin-mediated endothelial P-selectin translocation: Cell signaling mechanisms. J. Leukoc. Biol. 83:352-360.

Mofarrahi, M., T. Nouh, S. Qureshi, L. Guillot, D. Mayaki, and S. N. A. Hussain. 2008. Regulation of angiopoietin expression by bacterial lipopolysaccharide. Am. J. Physiol. Lung Cell. Mol. Physiol. 294:L955-L963.

Nalimov, V. V. 1963. The Application of Mathematical Statistics to Chemical Analysis. Pergamon Press, Oxford, UK. 
Pfaffl, M. W. 2001. Development and validation of an externally standardised quantitative insulin like growth factor-1 (IGF-1) RT-PCR using LightCycler SYBR ${ }^{\circledR}$ Green I technology. Pages 281-292 in Rapid cycle real-time PCR, methods and applications. S. Meuer, C. Wittwer, and K. Nakagawara, ed. Springer, Heidelberg, Germany.

R Core Team. 2012. R: A Language and Environment for Statistical Computing. R Foundation for Statistical Computing, Vienna, Austria.

Rupp, R., and D. Boichard. 2001. Phenotypic and genetic relationships between milk somatic cell counts and clinical mastitis in the French Holstein cattle population. Prod. Anim. 14:193-200.

Sako, D., X.-J. Chang, K. M. Barone, G. Vachino, H. M. White, G. Shaw, G. M. Veldman, K. M. Bean, T. J. Ahern, B. Furie, D. A. Cumming, and G. R. Larsen. 1993. Expression cloning of a functional glycoprotein ligand for P-selectin. Cell 75:1179-1186.

Scholz, A., V. Lang, R. Henschler, M. Czabanka, P. Vajkoczy, E. Chavakis, J. Drynski, P. N. Harter, M. Mittelbronn, D. J. Dumont, K. H. Plate, and Y. Reiss. 2011. Angiopoietin-2 promotes myeloid cell infiltration in a $\beta_{2}$-integrin-dependent manner. Blood 118:5050-5059.

Sturn, D. H., C. Feistritzer, B. A. Mosheimer, A. Djanani, K. Bijuklic, J. R. Patsch, and C. J. Wiedermann. 2005. Angiopoietin affects neutrophil migration. Microcirculation 12:393-403.
Suri, C., P. F. Jones, S. Patan, S. Bartunkova, P. C. Maisonpierre, S. Davis, T. N. Sato, and G. D. Yancopoulos. 1996. Requisite role of angiopoietin-1, a ligand for the TIE2 receptor, during embryonic angiogenesis. Cell 87:1171-1180.

Tetens, J., J. J. Friedrich, A. Hartmann, M. Schwerin, E. Kalm, and G. Thaller. 2010. The spatial expression pattern of antimicrobial peptides across the healthy bovine udder. J. Dairy Sci. 93:775-783.

Thomsen, H., N. Reinsch, N. Xu, C. Looft, S. Grupe, C. Kuhn, G. A. Brockmann, M. Schwerin, B. Leyhe-Horn, S. Hiendleder, G. Erhardt, I. Medjugorac, I. Russ, M. Förster, B. Brenig, F. Reinhardt, R. Reents, J. Blümel, G. Averdunk, and E. Kalm. 2000. A male bovine linkage map for the ADR granddaughter design. J. Anim. Breed. Genet. 117:289-306.

Warnes, G., and F. Leisch. 2012. Population Genetics. R package. http://cran.r-project.org/web/packages/genetics/.

Ye, S., S. Dhillon, X. Ke, A. R. Collins, and I. N. M. Day. 2001. An efficient procedure for genotyping single nucleotide polymorphisms. Nucleic Acids Res. 29:e88.

You, F. M., N. Huo, Y. Q. Gu, M. Luo, Y. Ma, D. Hane, G. R. Lazo, J. Dvorak, and O. D. Anderson. 2008. BatchPrimer3: A high throughput web application for PCR and sequencing primer design. BMC Bioinformatics 9:253. 